\title{
Techniques for comprehensive two dimensional echocardiographic assessment of left ventricular systolic function
}

\author{
T H Marwick
}

Heart 2003;89(Suppl III):iii2-iii8

$T$ he quantitation of left ventricle (LV) volumes and ejection fraction is an important aspect of cardiac evaluation in all cardiac disorders. Prognosis in many types of heart disease is closely related to global left ventricular ejection fraction (LVEF), falling off rapidly as the ejection fraction falls below $40 \%$ (fig 1). However, although ejection fraction has the advantage of being a simple numerical parameter that reflects LV function, it is strongly influenced by loading conditions and does not correlate well with symptom status. Perhaps more importantly, although two dimensional (2D) ejection fraction is meaningful when applied across populations or to stratify risk in individuals, its value as a sequential test within individuals is constrained by limited test-retest reliability.

\section{GLOBAL FUNCTION ASSESSMENT BY 2D ECHOCARDIOGRAPHY \\ Subjective assessment}

Whatever the limitations of subjective assessment, the reality is that echocardiographic assessment of global left ventricular systolic function is usually performed subjectively. Moreover, the eye of an experienced observer is comparable to trackball measurements. ${ }^{1}$ The situations where this approach can be misleading are when the rhythm is irregular (when examining a long tape run rather than individual cine loops is essential), the LV size is very large or very small, and at the extremes of heart rate.

\section{Measurement of ventricular volumes and ejection fraction}

Two dimensional echocardiography approaches for calculation of LV volumes have largely superseded $M$ mode echocardiography techniques that used geometric assumptions based on the minor dimension of the ventricle. A number of 2D approaches have been described (table 1), ${ }^{2-4}$ some using more sophisticated geometric assumptions. With each of these methods, once volumes have been measured, ejection fraction is simply measured as (LVEDV LVESV)*100/LVEDV.

Modified Simpson's rule

This method is based on disc summation, analogous to examining a stack of coins. ${ }^{5}$ Provided a sufficient number of discs are measured, this method will overcome nongeometric bulges in the longitudinal axis, although the technique is not truly "non-geometric", as each disc is expected to be circular, ignoring the dimension in other than the measured plane. The definition and calculation of each disc is automatically performed by the ultrasound machine software after the sonographer defines the central axis of the LV cavity and traces the borders. Biplane data acquisition (apical four and two chamber views) is desirable in order to overcome this, although this is sometimes not possible because of inadequate endocardial resolution of the anterior wall or foreshortening in the apical two chamber view. The usual rule is that views should not be combined if the chamber length calculations are different by $>20 \%$.

\section{Area-length method}

This method is appropriate in symmetrical LV cavities and is usually applied using the apical four chamber view. The volume is derived from the area of the LV squared, divided by length and multiplied by 0.85 , to reflect the non-cylindrical shape of the apex. This has a number of shortcomings related to non-geometric LV shapes and remodelling (which may change the LV shape from a cylinder to a sphere).

\section{Geometric methods}

These involve some combination between the shape in the short axis view and ventricular length, or combined geometric figures including combinations of cylinders, truncated cones, and cones. These have been superseded by the high feasibility of the Simpson's method.

\section{Volumetric approaches}

Although the results with 2D echo have been useful for categorising risk within populations, the limited test-retest reproducibility of 2D imaging poses problems for the application of this test on a sequential basis within an individual. The topic of 3D imaging is outside the ambit of this review, but it should be recognised that more accurate and reproducible measurements have been obtained using three dimensional (3D) techniques. ${ }^{6}$ Previously, 3D echocardiography was constrained by the need to reconstruct a 3D dataset from a number of 2D images, identified in direction and position relative to a fixed frame of reference. Recently, real time 3D imaging has become available, and in conjunction with offline edge detection programs (fig 2) it is likely that this approach will become the standard for LV volume and ejection fraction measurement with echocardiography. ${ }^{78}$

\section{Exercise ejection fraction}

While also out of the remit of this review, exercise 2D echocardiography may be useful for the identification of subclinical LV dysfunction-for example, in valvar heart disease. In this situation, standard measurements (including Simpson's rule) have been used to measure the LV contractile reserve. ${ }^{9}$

Abbreviations: $B N P$, type $B$ natriuretic peptide; $C A D$, coronary artery disease; $E B C T$, electron beam computed tomography LV, left ventricle; LVEF, left ventricular ejection fraction; SPECT, single photon emission computed tomography 


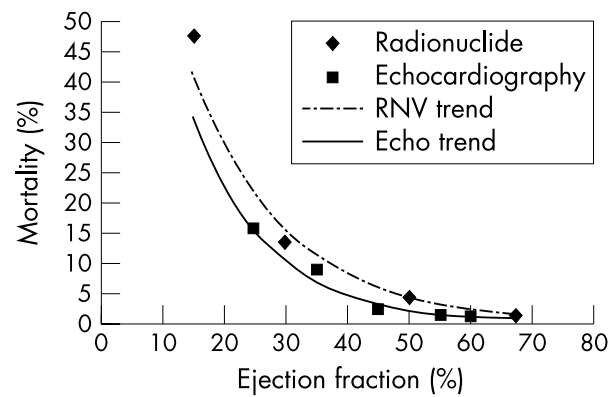

Figure 1 Mortality after measurement of ejection fraction by radionuclide ventriculography (one year follow up, Multicentre Postinfarction Research Group, 1983) and after 2D echo measurement of ejection fraction (six month follow up, GISSI study 1993). Note the close correlation of the curves, allowing for the difference in follow up between the two cohorts.

\section{Load independent techniques}

Assessment of LV systolic function is usually performed in order to gather insight about the contractile state of the left ventricle. The problem is that performance is dependent on not only contractile state but also load. Thus, while image quality and geometric assumptions pose important practical limitations on the use of 2D echo ejection fraction as a clinical tool, the major limitation is the dependence of ejection fraction on loading conditions and heart rate (fig 3 ). There are two ways of addressing this: to measure afterload and preload in order to correct the ejection fraction, or to try to measure contractility independent of loading.

\section{Measurement of load}

Load determines wall stress $(\sigma)$, which is defined by force over each unit of cross sectional area; this force being determined by pressure (P). Afterload is reflected by systolic wall stress. Cuff systolic pressure may overestimate LV systolic pressure (due to wave reflection), so some authors have used mean arterial pressure, which is usually equivalent centrally and peripherally. As LV diastolic pressure cannot be accurately determined non-invasively, wall stress parameters
Table 1 Normal values of LV volumes by 2D echocardiography. Variation of ejection fraction is $7-10 \%^{2-4}$

\begin{tabular}{lrll}
\hline & Men & Women & $95 \% \mathrm{Cl}$ \\
\hline Biplane Simpson's & $111(22)$ & $80(12)$ & $11 \%$ \\
EDV & $34(12)$ & $29(10)$ & $15 \%$ \\
ESV & $112(27)$ & $89(20)$ & $15 \%$ \\
Area length (A4C) & $35(16)$ & $25(12)$ & $25 \%$ \\
EDV & $130(27)$ & $92(19)$ & \\
ESV & $40(14)$ & $31(11)$ & \\
Area length (A2C) & &
\end{tabular}

Values are mean (SD)

$\mathrm{Cl}$, confidence interval; EDV, end diastolic volume, ESV, end systolic volume.

cannot be used to measure preload, the closest analogue of which is diastolic volume.

Meridional stress relates to the load posed by long axis shortening (that is, base-apex), and is calculated as $\sigma \mathrm{m}=\mathrm{P} \times \mathrm{LVID} /[4 \times \mathrm{Th}(1-\mathrm{LVID} / \mathrm{Th})]$, where $\mathrm{P}$ (pressure) is approximated to systolic blood pressure, LVID (cavity dimension) is end systolic dimension, and Th is average wall thickness. ${ }^{10}$ Circumferential stress reflects stress in the minor axis; $\sigma c=\mathrm{P} \times \mathrm{a}^{2}\left[\mathrm{l}+\left(\mathrm{b}^{2} / \mathrm{r}^{2}\right)\right] /\left(\mathrm{b}^{2}-\mathrm{a}^{2}\right)$, where $\mathrm{a}$ is the internal radius, $b$ is the epicardial radius, and $r$ is the midwall radius. ${ }^{11}$ Both stress measurements can be measured with dimensions acquired with $2 \mathrm{D}$ echo, or using areas from 2D echo.

\section{Load corrected parameters}

All clinical parameters of LV function are load dependent. The problem with trying to isolate contractility is that alterations of loading result in length dependent changes in contractility, independent of the Frank-Starling mechanism.

LV midwall shortening expresses the stress shortening relation of the ventricle. The use of midwall shortening is less dependent on LV geometry than are endocardial measurements.

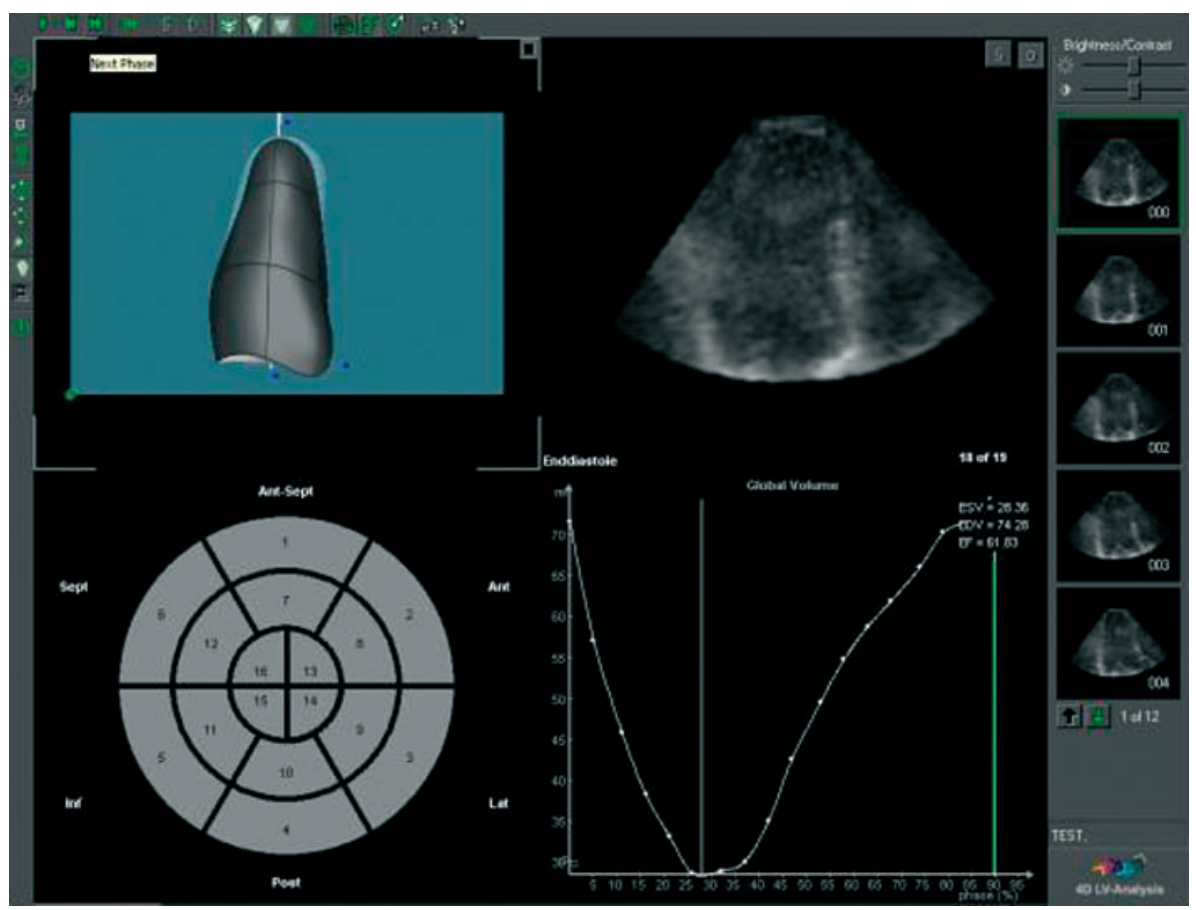

Figure 2 Three dimensional (3D) echocardiographic evaluation of left ventricular (LV) volumes and ejection fraction. Images from a volumetric dataset are reconstructed (upper centre and right column) and semi-automated edge detection is used to trace the endocardial border and construct a 3D model in systole and diastole (upper left). Expression of volumes in each image yields a time-volume curve (lower left) from which ejection fraction is calculated. 

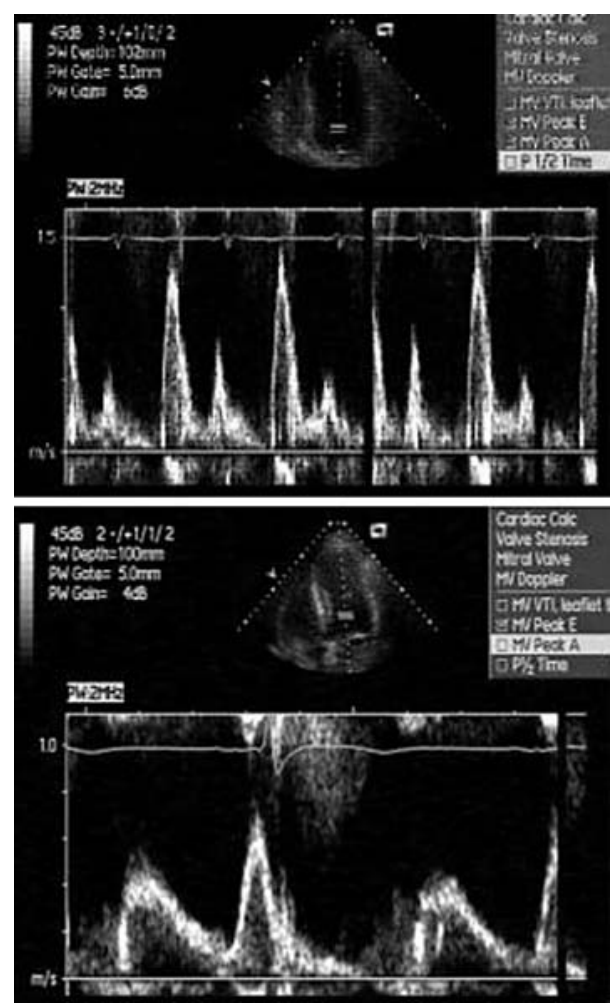

Figure 3 Dependence of LV volumes and standard Doppler indices of diastolic function on loading conditions. This patient with renal failure was studied before (above) and after dialysis (below). LV size and LV filling patterns are altered by loading.

Pressure-volume loops are the standard approach to expressing the association between loading and inotropic state. The end systolic pressure-volume relation is drawn in a sequence of these curves under different inotropic states, to generate end systolic elastance. Other authors have charted LV wall stress against LV dimension. ${ }^{12}$ An increase in the slope of this curve reflects increasing contractile state, and a decrease corresponds to depressed contractility. The development of automated LV volume measurement techniques has been used to apply this approach non-invasively,,$^{13}$ but the AQ technique has some limitations related to image quality and beat-to-beat variations of LV volume may be large, reflecting the impact of different cut planes with respiration, for example. This approach has therefore not been widely applied for clinical research.

Preload recruitable stroke work has been used in animal models for over 15 years. ${ }^{14}$ These investigators found that the relation between stroke work and end diastolic segment length or chamber volume (termed the preload recruitable stroke work relation) was highly linear. This contrasts with the use of filling pressure as the marker of preload, when a curvilinear function is obtained that plateaus at higher filling pressures. ${ }^{14}$ The problems of using this clinically are in accurately measuring stroke work (which requires accurate knowledge of central aortic pressure) and LV volume (the limitations of 2D echo for which are discussed above), and for altering loading without altering inotropy - in experimental settings, these steps have included balloon occlusion of the vena cava. For practical reasons, this technique has not attained everyday use.

Alternative and less load dependent measures include the peak systolic pressure-end systolic volume ratio ${ }^{15}$ and cardiac power. ${ }^{16}$
Cardiac power correlates with $\mathrm{Vo}_{2}$ max and predicts prognosis, but has previously been measured invasively. Mean power is calculated from the product of stroke volume, mean arterial pressure, and heart rate, and peak instantaneous power is the peak instantaneous product of left ventricular outflow and pressure during systole. ${ }^{16}{ }^{17}$ Either parameter may be derived using Doppler echocardiography to measure stroke volume, and 2D echocardiography is used to calculate "preload adjusted" power (corrected for end diastolic volume)..$^{18}$ Nonetheless, this is not strictly a 2D echocardiography technique.

\section{Complementary techniques}

Doppler may be used to measure LV ejection. However, the measurement of stroke volume is dependent on the accuracy of LV outflow tract measurement, errors of which are squared in the course of volume calculations. The measurement of LV $\mathrm{dP} / \mathrm{dt}$ is a relatively load independent marker of LV contractility, which is especially valuable in mitral regurgitation, when contractility may be overestimated by the ejection fraction. The myocardial performance index is derived from the sum of the isovolumic contraction time and isovolumic relaxation time, divided by the ejection time. This measurement is reproducible, easily obtainable, and correlated closely with invasive measures of both systolic and diastolic function. ${ }^{19}$ It appears to be independent of geometry, although it remains somewhat load dependent. Finally, tissue Doppler techniques have the benefit of being less dependent on image quality than 2D imaging, and do not require tracing; both annular displacement ${ }^{20}$ and average velocity ${ }^{21}$ have been correlated with ejection fraction.

\section{REGIONAL FUNCTION ASSESSMENT BY 2D ECHOCARDIOGRAPHY}

\section{Subjective assessment}

The qualitative evaluation of LV systolic function is based on the division of the LV into a number of segments, after which each segment is scored as normal, hypokinetic, akinetic, or dyskinetic. The main problems pertain to the distinction of hypokinesia from akinesia. We judge akinesia to be present when endocardial excursion is $<2 \mathrm{~mm}$, and hypokinesia with endocardial excursion $<5 \mathrm{~mm}$. However, movement may be passive, and thickening is the more reliable marker of contractility.

The standard 16 segment model of the American Society of Echocardiography (septal, lateral, anterior, and inferior at the apex, with these segments as well as anteroseptal and posterior segments at the base and mid papillary muscle level) (fig 4) is likely to remain in widespread use because the suggested 17 segment model (which includes a true apical segment) ignores the small but important detail that most echocardiograms fail to identify the true apex of the heart. Our laboratory uses a modification of the American Society of Echocardiography segmentation. ${ }^{22}$ With this, a score of 1 is given for normal regions, with scores of 2, 3, and 4 for hypokinesis, akinesis, and dyskinesis, 5 for aneurysm, and 6 and 7 for akinesis or dyskinesis with thinning, respectively. The wall motion "score index" (obtained by averaging the scores of individual segments), gives a semi-quantitative index of global systolic function, analogous to the ejection fraction and with similar prognostic significance. ${ }^{23}$

Regional wall motion scoring is highly reproducible within individual sites, ${ }^{24}$ reflecting common reading styles. However, reproducibility of wall motion assessment between centres may be quite limited, especially during stress 2D echo. ${ }^{25}$ Concordance may be improved with the use of standard reading criteria ${ }^{26}$ and harmonic imaging. ${ }^{27}$ While it is unlikely that echocardiographers will stop visually assessing the LV, an objective measure that supplemented this assessment 

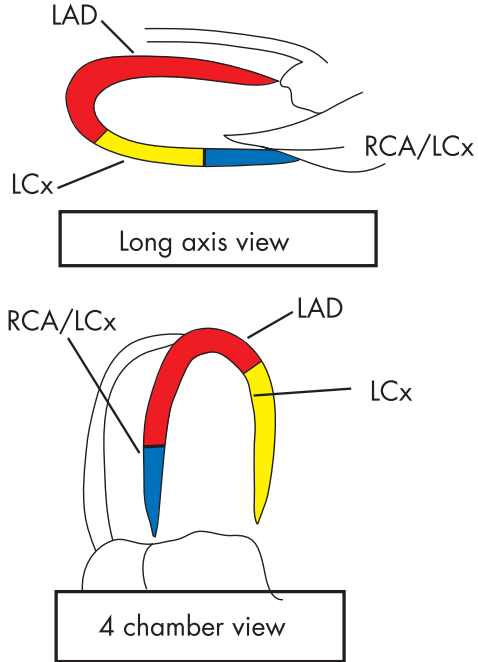

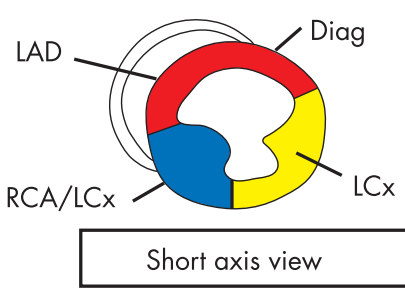

Figure 4 The 16 segment American Society of Echocardiography model for characterisation of regional LV function, and usual coronary artery distribution of the segments. Reproduced from: Marwick TH. Stress echocardiography - its role in the diagnosis and evaluation of coronary artery disease. Boston: Kluwer Academic Publishers, 2003, with permission of the publisher.

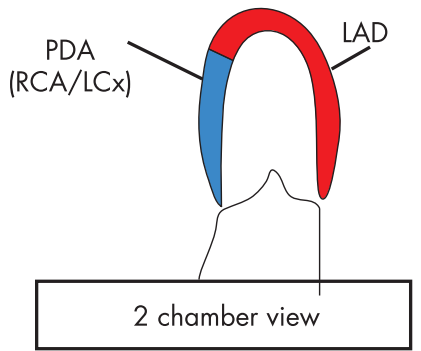

1 = normal; 2 = hypokinetic; 3 = akinetic; 4 =dyskinetic

with objective criteria could act as a "common language" to reduce variation between readers.

\section{Objective assessment}

A number of echo and Doppler modalities are able to offer quantitation of regional function, ${ }^{28}$ and most are outside the remit of this review (table 2 ). The $2 \mathrm{D}$ echo based techniques include techniques for assessing radial displacement (using the centre line method or colour kinesis) or thickening (anatomical M mode).

\section{Centre line method}

This method is based upon three steps: tracing the LV end diastolic and end systolic borders; superimposition of the traces with interpolation of the centre line; and measurement of the excursion from this line in a series of chords perpendicular to the centre line, which can be compared to a normal range of displacement. Each step poses potential pitfalls.

Tracing of contours, preferably in two orthogonal planes (usually apical four and two chamber views) is dependent on good quality border definition, and the reliance on apical views may compromise edge detection because of the parallel orientation of the echocardiographic beam with the endocardium. Although good border definition has become more available with the development of harmonic and contrast imaging, tracing the edge may still need an element of guesswork. More than a single frame may need to be traced at both systole and diastole, making the procedure time

Table 2 Alternative techniques for the quantification of regional LV function ${ }^{28}$

\begin{tabular}{|c|c|c|}
\hline & Radial & Longitudinal \\
\hline Displacement & $\begin{array}{l}\text { Centre line (from 2D echo) } \\
\text { Colour kinesis }\end{array}$ & $\begin{array}{l}\text { Annular } \mathrm{M} \text { mode } \\
\text { Tissue tracking }\end{array}$ \\
\hline Thickening & $\begin{array}{l}\text { Anatomical M mode } \\
\text { Integrated backscatter }\end{array}$ & \\
\hline Velocity & $\begin{array}{l}\text { Velocity from displacement } \\
\text { Longitudinal velocity } \\
\text { Tissue Doppler gradient }\end{array}$ & $\begin{array}{l}\text { Tissue Doppler velocity } \\
\text { Strain }\end{array}$ \\
\hline Timing & $\begin{array}{l}\text { Time to peak systole } \\
\text { Time to onset of diastole }\end{array}$ & $\begin{array}{l}\text { Time to peak systole } \\
\text { Time to onset of diastole }\end{array}$ \\
\hline
\end{tabular}

consuming, although automated and semi-automated methods of tracking the wall have been developed (fig 5).

The superimposition of systole and diastole may have a critical effect on the measurement of excursion from the centre line. Either fixed or floating frames of reference can be used to compensate for rotational or translational movement of the heart. ${ }^{29}$ Failure to correct for such movements may cause false positives, but the use of correction may hinder the detection of milder abnormalities. Finally, different variations of the technique measure the chords relative to the centre line or relative to the centre of LV mass.

\section{Colour kinesis method}

The colour kinesis method uses acoustic quantification to define the border, based on the difference in backscatter between the LV wall and cavity. This has the benefit of avoiding the onerous process of tracing the border in every frame, although the frame rate is somewhat limited, compared to standard 2D imaging. The excursion of the myocardium from each frame to the next is filled with a different colour, and the resulting display overlaid on the 2D image ("colour kinesis"). ${ }^{30}$ The displacement is portrayed as segmental area shrinkage, ${ }^{31}$ and arranged in stacked histograms (fig 6), which can be compared to normal ranges. This approach has been particularly applied during stress echo, where it correlates with expert wall motion analysis and may be of value to less expert readers. ${ }^{32}$

This technique is heavily dependent on image quality, and appears to be more feasible with the use of myocardial contrast for LV opacification. ${ }^{33}$ Measurements show a variation of $10-20 \%$. As with any technique that measures endocardial motion, this is sensitive to extrinsic cardiac movement.

\section{Anatomic $M$ mode}

Myocardial thickening is the optimal parameter for measurement, because unlike excursion, it is independent of cardiac rotation or translation. However, the measurement of thickening requires definition of both the endocardium and the epicardium-and the latter can pose a problem in the apical views. M mode ultrasound has conventionally been used for gathering wall thickening data, but has been constrained by the angle dependence of standard $\mathrm{M}$ mode imaging. Two dimensional images at high temporal and spatial resolution have been used to reconstruct $M$ mode 

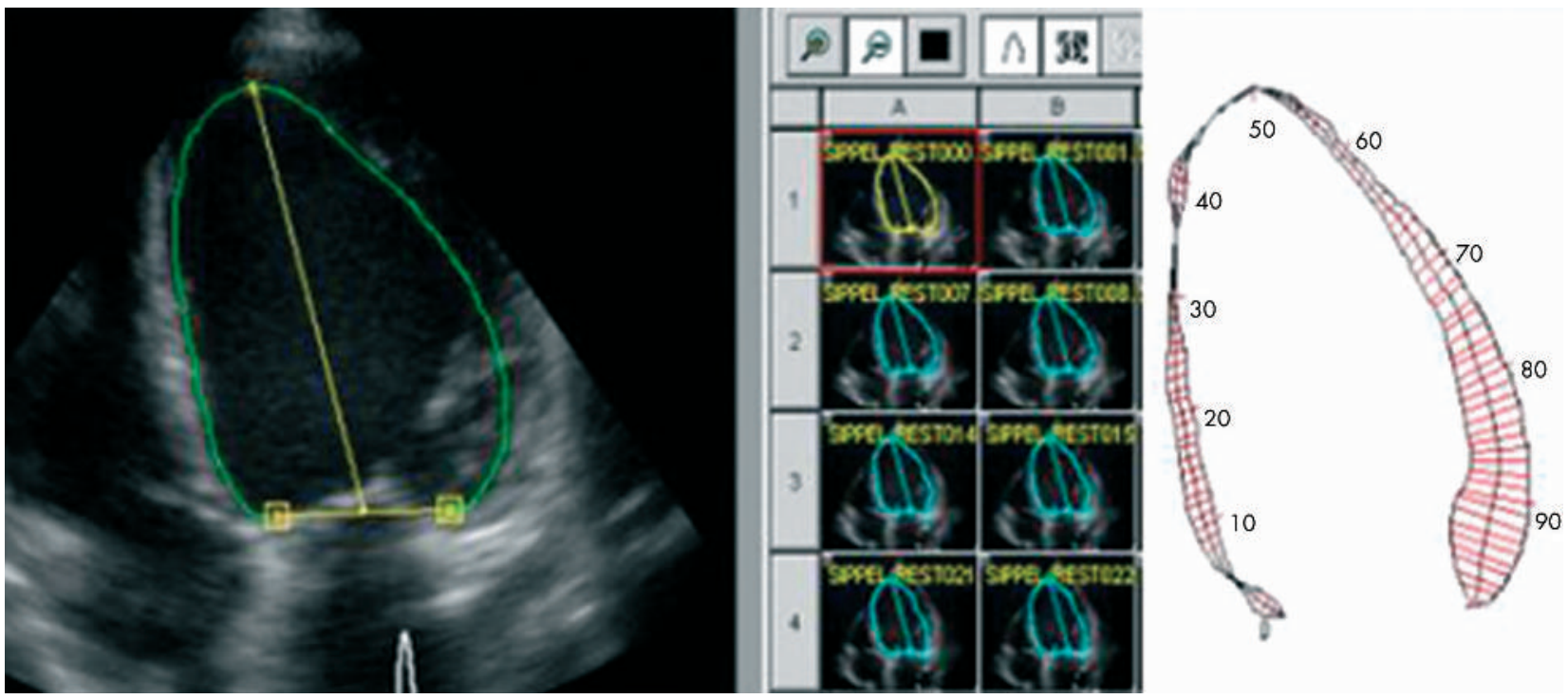

Figure 5 Centre line approach to quantification of regional LV function. Automated detection of the endocardial border is applied in each view (left), repeated in each frame (thumbprint images, centre), and regional excursion between end diastole and end systole is measured using the centre line method. Reproduced from: Marwick TH. Stress echocardiography - its role in the diagnosis and evaluation of coronary artery disease. Boston: Kluwer Academic Publishers, 2003, with permission of the publisher.

images in any plane "anatomic M-mode", ${ }^{34}$ although caution has to be applied with angle corrections of $>60-70^{\circ} .{ }^{35}$ The results correlate well with visual assessment but it has been difficult to designate a normal range, because of variations of baseline thickening, ${ }^{34}$ and the clinical benefit of this approach is not well defined.

\section{ALTERNATIVE APPROACHES}

Two dimensional echocardiography remains the most widely accepted technique for assessment of LV systolic function, reflecting its versatility and ability to identify complications (for example, thrombi) and associated problems (for example, mitral regurgitation). Nonetheless, other modalities may represent alternatives or may even be used to select patients for $2 \mathrm{D}$ echo.

\section{Clinical evaluation}

The clinical signs of LV dysfunction are insensitive-for example, in 14507 patients in the CASS registry, a third heart sound or crackles had a respective sensitivity of $9 \%$ and $5 \%$ for the detection of significant LV dysfunction on contrast ventriculography. ${ }^{36}$ Part of this low sensitivity is caused by

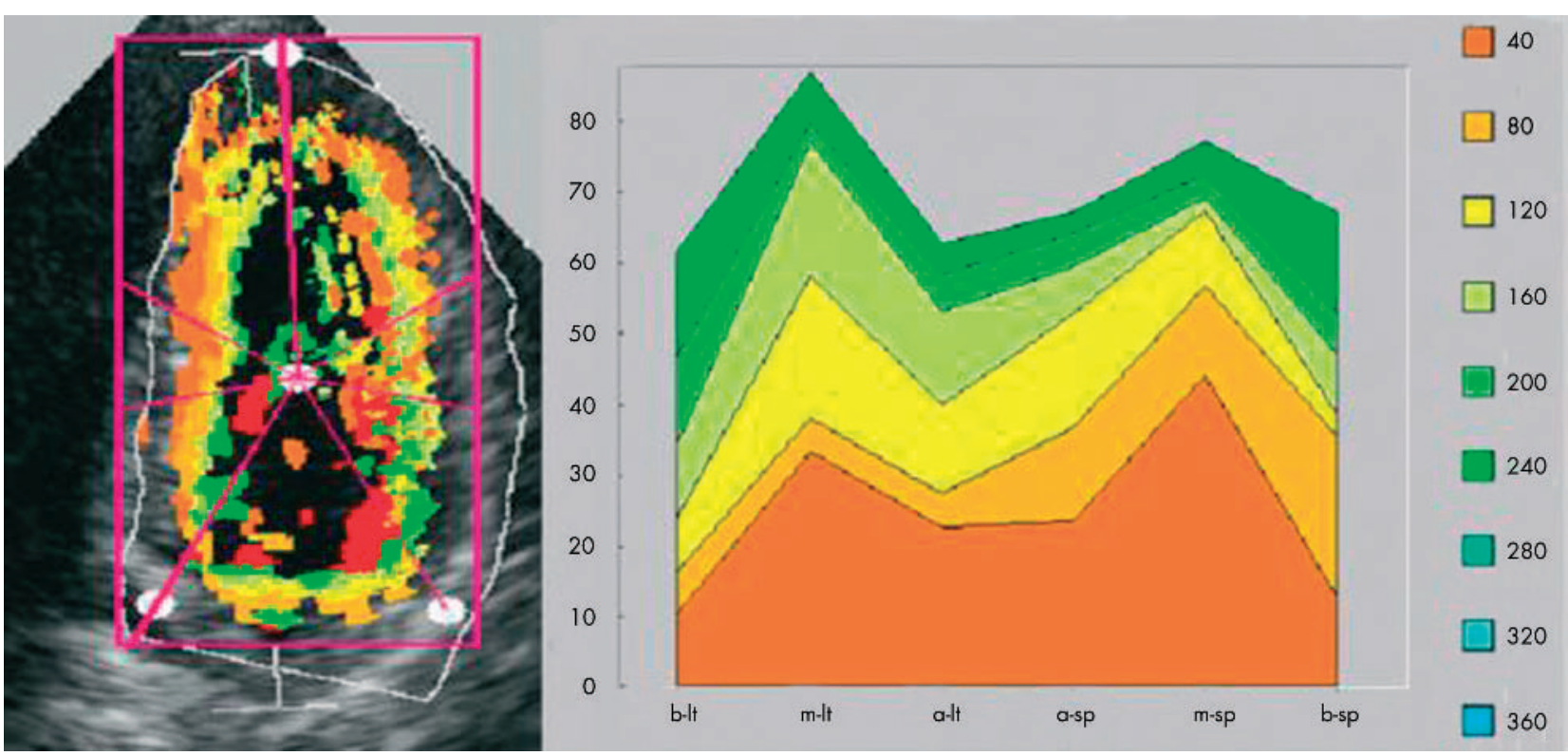

Figure 6 Quantitation of radial function using colour kinesis. Each successive ultrasound frame on the image (leff) is coded with a different colour. The histogram (right) shows the fractional area change within each segment (x axis). Reproduced from: Marwick TH. Stress echocardiography - its role in the diagnosis and evaluation of coronary artery disease. Boston: Kluwer Academic Publishers, 2003, with permission of the publisher. 
masking of the clinical signs by treatment. Moreover, the signs and symptoms of LV systolic and predominantly diastolic dysfunction are indistinguishable.

The limitations of the clinical exam are concerning from a health economy standpoint. Fortunately, the standard 12 lead ECG may be used to identify patients with possible LV dysfunction. ${ }^{37}{ }^{38}$ A completely normal ECG, or even narrow QRS complexes, has a high negative predictive value for excluding significant LV dysfunction. ${ }^{39}$ Thus, a normal ECG could assist triage of patients referred for echocardiography. ${ }^{40}$ Indeed, a normal cardiothoracic ratio measured in the posteroanterior chest $x$ ray does not add significantly to the value of a normal ECG in predicting normal systolic function. ${ }^{36}$

\section{Type B natriuretic peptide (BNP)}

The clinical signs of LV dysfunction are insensitive, and the detection of LV dysfunction by echocardiography in all "at risk" patients is prohibited by cost and availability. Although BNP has been shown to be a useful marker of systolic dysfunction in symptomatic patients, ${ }^{41}$ the ability of this test to act as a screening tool for subclinical disease appears limited. ${ }^{42}$

\section{Nuclear ventriculography}

Nuclear techniques include first pass ventriculography, equilibrium RNV (gated blood pool scanning), and gated single photon emission computed tomography (SPECT), during myocardial perfusion scanning with thallium-201 or $\mathrm{Tc}^{99 \mathrm{~m}}$-sestamibi. ${ }^{43}{ }^{44}$ After myocardial infarction, LVEF measured by these tests has been correlated with prognosis, in both the pre-thrombolytic ${ }^{45}$ and thrombolytic eras, ${ }^{46}$ and is incremental to clinical indicators of prognosis. ${ }^{47}$ Reduction of exercise LVEF is an indicator of severe coronary artery disease $(\mathrm{CAD}),{ }^{48}{ }^{49}$ but not a specific marker of CAD. ${ }^{50}$ Nonetheless, peak exercise LVEF by gated SPECT provides incremental prognostic value to SPECT perfusion imaging. ${ }^{51}$

\section{Computed $x$ ray tomography}

Electron beam computed tomography (EBCT) permits precision measurement of cardiac structure and function, from images acquired during a single cardiac cycle. EBCT has been validated against contrast ventriculography ${ }^{52}$ and radionuclide ventriculography. ${ }^{53}$ There are limited data using EBCT derived LV function to predict cardiac risk in clinical practice. Conventional CT has previously lacked the spatial or temporal resolution to permit measurement of LV volumes and function, but ECG gating and other developments have made this more feasible.

\section{Cardiac magnetic resonance imaging (MRI)}

Cine MRI has excellent temporal and spatial resolution, and image plan reproduction is much higher than with 2D echo because it can image in any plane. Cardiac MRI has become the in vivo "gold standard" for LV volumes and function assessment, and has been validated against contrast ventriculography, ${ }^{54}$ radionuclide ventriculography ${ }^{55}$ and echocardiography. ${ }^{56}$ The excellent test-retest reliability of this technique has enabled much smaller sample sizes for research studies with MRI than $2 \mathrm{D}$ echo, ${ }^{57}$ but its use as a routine clinical tool is constrained by cost, availability, and expertise.

\section{CONCLUSION}

Despite its limitations, 2D echocardiography remains the most widely used non-invasive technique for clinical assessment of LV systolic function, and is likely to remain so because it is non-invasive, inexpensive, and widely available. LV systolic measurements are dependent on loading conditions, and assessment of loading should be considered in the interpretation of ejection fraction. Problems with accuracy and reproducibility of volumes and ejection fraction pertain largely to the geometric challenges of 2D imaging, and are likely to be solved by 3D approaches.

Correspondence to: Professor Thomas H Marwick, University of Queensland Department of Medicine, Princess Alexandra Hospital, Brisbane, Qld 4102, Australia; tmarwick@medicine.pa.uq.edu.au

\section{REFERENCES}

1 Foster $\mathbf{E}$, Cahalan MK. The search for intelligent quantitation in echocardiography: "eyeball," "trackball" and beyond. J Am Coll Cardiol 1993;22:848-50

2 Wyatt HL, Heng MK, Meerbaum S, et al. Cross-sectional echocardiography. II. Analysis of mathematic models for quantifying volume of the formalin-fixed left ventricle. Circulation 1980;61:1119-25.

3 Gordon EP, Schnittger I, Fitzgerald PJ, et al. Reproducibility of left ventricular volumes by two-dimensional echocardiography. J Am Coll Cardiol 1983;2:506-13.

4 Himelman RB, Cassidy MM, Landzberg JS, et al. Reproducibility of quantitative two-dimensional echocardiography. Am Heart J 1988; 115:425-31

5 Stamm RB, Carabello BA, Mayers DL, et al. Two-dimensional echocardiographic measurement of left ventricular ejection fraction: prospective analysis of what constitutes an adequate determination. Am Heart J 1982;104:136-44.

6 Chuang ML, Hibberd MG, Salton CJ, et al. Importance of imaging method over imaging modality in noninvasive determination of left ventricular volumes and ejection fraction: assessment by two- and three-dimensional echocardiography and magnetic resonance imaging. J Am Coll Cardiol 2000;35:477-84

7 Pearlman AS. Measurement of left ventricular volume by three-dimensional echocardiography - present promise and potential problems [editorial; comment]. J Am Coll Cardiol 1993;22:1538-40.

8 King DL, Harrison MR, King DL, Jr, et al. Improved reproducibility of left atrial and left ventricular measurements by guided three-dimensional echocardiography. J Am Coll Cardiol 1992;20:1238-45.

9 Leung DY, Griffin BP, Stewart WJ, et al. Left ventricular function after valve repair for chronic mitral regurgitation: predictive value of preoperative assessment of contractile reserve by exercise echocardiography. J Am Coll Cardiol 1996;28: 1 198-205.

10 Reichek N, Wilson J, St John SM, et al. Noninvasive determination of left ventricular end-systolic stress: validation of the method and initial application. Circulation 1982;65:99-108.

11 Gaasch WH, Zile MR, Hoshino PK, et al. Stress-shortening relations and myocardial blood flow in compensated and failing canine hearts with pressure-overload hypertrophy. Circulation 1989;79:872-83.

12 Aurigemma GP, Gaasch WH, Villegas B, et al. Noninvasive assessment of left ventricular mass, chamber volume, and contractile function. Curr Prob Cardiol 1995;20:361-440.

13 Denault AY, Gorcsan J III, Mandarino WA, et al. Left ventricular performance assessed by echocardiographic automated border detection and arterial pressure. Am J Physiol 1997;272:H138-47

14 Glower DD, Spratt JA, Snow ND, et al. Linearity of the Frank-Starling relationship in the intact heart: the concept of preload recruitable stroke work. Circulation 1985;71:994-1009.

15 Ginzton LE, Laks MM, Brizendine M, et al. Noninvasive measurement of the rest and exercise peak systolic pressure/end-systolic volume ratio: a sensitive two-dimensional echocardiographic indicator of left ventricular function. J Am Coll Cardiol 1984:4:509-16.

16 Armstrong GP, Carlier SG, Fukamachi K, et al. Estimation of cardiac reserve by peak power: validation and initial application of a simplified index. Heart 1999:82:357-64.

17 Tan LB. Evaluation of cardiac dysfunction, cardiac reserve and inotropic response. Postgrad Med J 1991;67(suppl 1):S10-20.

18 Mandarino WA, Pinsky MR, Gorcsan J III. Assessment of left ventricular contractile state by preload-adjusted maximal power using echocardiographic automated border detection. J Am Coll Cardiol 1998:31:861-8.

19 Tei C, Nishimura RA, Seward JB, et al. Noninvasive Doppler-derived myocardial performance index: correlation with simultaneous measurements of cardiac catheterization measurements. J Am Soc Echocardiogr 1997: 10:169-78.

20 Pan C, Hoffmann R, Kuhl H, et al. Tissue tracking allows rapid and accurate visual evaluation of left ventricular function. Eur J Echocardiogr 2001;2: 197-202

21 Haluska BA, Short L, Marwick TH. Relationship of ventricular longitudinal function to contractile reserve in patients with mitral regurgitation. Am Heart $J$ 2003;146:183-8.

22 Broderick T, Sawada S, Armstrong WF, et al. Improvement in rest and exercise-induced wall motion abnormalities after coronary angioplasty: an exercise echocardiographic study. J Am Coll Cardiol 1990;15:591-9.

23 Nishimura RA, Reeder GS, Miller FA, et al. Prognostic value of predischarge 2-dimensional echocardiogram after acute myocardial infarction. Am J Cardiol 1984;53:429-32.

24 Oberman A, Fan PH, Nanda NC, et al. Reproducibility of two-dimensional exercise echocardiography. J Am Coll Cardiol 1989;14:923-8. 
25 Hoffmann R, Lethen $\mathrm{H}$, Marwick T, et al. Analysis of interinstitutional observer agreement in interpretation of dobutamine stress echocardiograms. J Am Coll Cardiol 1996;27:330-6

26 Hoffmann R, Lethen H, Marwick T, et al. Standardized guidelines for the interpretation of dobutamine echocardiography reduce interinstitutional variance in interpretation. Am J Cardiol 1998;82:1520-4.

27 Hoffmann R, Poldermans D, van der Meer P, et al. The maturing of stress echocardiography: improved intercenter agreement in interpretation of dobutamine stress echocardiograms using new techniques. Eur Heart $J$ 2002;23:821-9.

28 Marwick TH. Quantitative techniques for stress echocardiography: dream or reality? Eur J Echocardiogr 2002;3:171-6.

29 Force TL, Folland ED, Aebisher N, et al. Echocardiographic assessment of left ventricular function. In: Marcus ML, Schelbert HR, Skorton DJ, Wolf GL, eds. Cardiac imaging. Philadelphia: WB Saunders, 1991.

30 Bednarz J, Vignon P, Mor-Avi V, et al. Color kinesis: principles of operation and technical guidelines. Echocardiography 1998;15:21-34.

31 Koch R, Lang RM, Garcia MJ, et al. Objective evaluation of regional left ventricular wall motion during dobutamine stress echocardiographic studies using segmental analysis of color kinesis images. J Am Coll Cardiol 1999;34:409-19.

32 Koch R, Lang RM, Garcia MJ, et al. Objective evaluation of regional left ventricular wall motion during dobutamine stress echocardiographic studies using segmental analysis of color kinesis images. J Am Coll Cardiol 1999;34:409-19.

33 Spencer KT, Bednarz J, Mor-Avi V, et al. Automated endocardial border detection and evaluation of left ventricular function from contrast-enhanced images using modified acoustic quantification. J Am Soc Echocardiogr 2002; 15:777-81.

34 Chan J, Wahi SCP, Marwick TH. Anatomical M-mode: a novel technique for the quantitative evaluation of regional wall motion analysis during stress echocardiography. Int J Card Imaging 2002;16:247-55.

35 Strotmann JM, Escobar Kvitting JP, Wilkenshoff UM, et al. Anatomic M-mode echocardiography: a new approach to assess regional myocardial function a comparative in vivo and in vitro study of both fundamental and second harmonic imaging modes. J Am Soc Echocardiogr 1999;12:300-7.

36 Rihal CS, Davis KB, Kennedy JW, et al. The utility of clinical, electrocardiographic, and roentgenographic variables in the prediction of left ventricular function. Am J Cardiol 1995;75:220-3.

37 Murkofsky RL, Dangas G, Diamond JA, et al. A prolonged QRS duration on surface electrocardiogram is a specific indicator of left ventricular dysfunction. J Am Coll Cardiol 1998:32:476-82.

38 O'Keefe JH Jr, Zinsmeister AR, Gibbons RJ. Value of normal electrocardiographic findings in predicting resting left ventricular function in patients with chest pain and suspected coronary artery disease. Am J Med 1989:86:658-62.

39 Davie AP, Francis CM, Love MP, et al. Value of the electrocardiogram in identifying heart failure due to left ventricular systolic dysfunction. BMJ 1996;312:222.

40 Talreja D, Gruver C, Sklenar J, et al. Efficient utilization of echocardiography for the assessment of left ventricular systolic function. Am Heart J 2000;139:394-8.

41 McCullough PA, Nowak RM, McCord J, et al. B-type natriuretic peptide and clinical judgment in emergency diagnosis of heart failure: analysis from breathing not properly (BNP) multinational study. Circulation 2002; 106:416-22.
42 Vasan RS, Benjamin EJ, Larson MG, et al. Plasma natriuretic peptides for community screening for left ventricular hypertrophy and systolic dysfunction: the Framingham heart study. JAMA 2002;288:1252-9.

43 Calnon DA, Kastner RJ, Smith WH, et al. Validation of a new counts-based gated single photon emission computed tomography method for quantifying left ventricular systolic function: comparison with equilibrium radionuclide angiography. J Nud Cardiol 1997;4:464-71.

$44 \mathrm{He}$ ZX, Cwajg E, Preslar JS, et al. Accuracy of left ventricular ejection fraction determined by gated myocardial perfusion SPECT with Tl-201 and Tc-99m sestamibi: comparison with first-pass radionuclide angiography. J Nucl Cardiol 1999;6:412-7

45 Multicenter Postinfarction Research Group. Risk stratification and survival after myocardial infarction. N Engl J Med 1983;309:331-6.

46 Zaret BL, Wackers FJ, Terrin ML, et al. Value of radionuclide rest and exercise left ventricular ejection fraction in assessing survival of patients after thrombolytic therapy for acute myocardial infarction: results of thrombolysis in myocardial infarction (TIMI) phase II study. The TIMl study group. J Am Coll Cardiol 1995;26:73-9

47 Griffin BP, Shah PK, Diamond GA, et al. Incremental prognostic accuracy of clinical, radionuclide and hemodynamic data in acute myocardial infarction. Am J Cardiol 1991;68:707-12.

48 Jones $\mathrm{RH}$, Johnson $\mathrm{SH}$, Bigelow $\mathrm{C}$, et al. Exercise radionuclide angiocardiography predicts cardiac death in patients with coronary artery disease. Circulation 1991;84:52-8.

49 Pryor DB, Harrell FE Jr, Lee KL, ef al. Prognostic indicators from radionuclide angiography in medically treated patients with coronary artery disease. Am J Cardiol 1984;53:18-22.

50 Haley JH, Miller TD, Christian TF, et al. Twelve-year outcome of patients with an abnormal exercise radionuclide left ventricular angiogram and angiographically insignificant coronary artery disease. Am J Cardiol 1998:82:418-22.

51 Sharir T, Germano G, Kavanagh PB, et al. Incremental prognostic value of post-stress left ventricular ejection fraction and volume by gated myocardial perfusion single photon emission computed tomography. Circulation 1999; 100:1035-42.

52 Rich S, Chomka EV, Stagl R, et al. Determination of left ventricular ejection fraction using ultrafast computed tomography. Am Heart J 1986;1 12:392-6.

53 Baik HK, Budoff MJ, Lane KL, et al. Accurate measures of left ventricular ejection fraction using electron beam tomography: a comparison with radionuclide angiography, and cine angiography. Int J Card Imaging 2000; 16:391-8.

54 Lawson MA, Blackwell GG, Davis ND, et al. Accuracy of biplane long-axis left ventricular volume determined by cine magnetic resonance imaging in patients with regional and global dysfunction. Am J Cardio 1996:77:1098-104.

55 Gaudio C, Tanzilli G, Mazzarotto P, et al. Comparison of left ventricula ejection fraction by magnetic resonance imaging and radionuclide ventriculography in idiopathic dilated cardiomyopathy. Am J Cardiol 1991;67:411-5.

56 Schalla $\mathrm{S}$, Nagel E, Lehmkuhl $\mathrm{H}$, et al. Comparison of magnetic resonance real-time imaging of left ventricular function with conventional magnetic resonance imaging and echocardiography. Am J Cardiol 2001;87:95-9.

57 Bottini PB, Carr AA, Prisant LM, ef al. Magnetic resonance imaging compared to echocardiography to assess left ventricular mass in the hypertensive patient. Am J Hypertens 1995;8:221-8. 